\title{
ORNITONIMIA ISTRIANA: \\ I NOMI POPOLARI DEL SUCCIACAPRE EUROPEO (CAPRIMULGUS EUROPAEUS)
}

0.

Il presente saggio tratta i nomi popolari della specie Caprimulgus europaeus (succiacapre europeo). Il succiacapre fa parte della famiglia dei Caprimulgidi (Caprimulgidae), ordine dei Caprimulgiformi (Caprimulgiformes). È presente più o meno in tutta la zona istro-quarnerina.

Vengono offerte soluzioni etimologiche ai nomi popolari raccolti dall'autore in Istria e sull'isola di Veglia. Gli ornitonimi presentati appartengono alle parlate istrovenete, istriote, istrorumene, croate e slovene.

I nomi delle località intervistate vengono riportati in italiano e in croato/sloveno; se la forma italiana non esiste, viene segnata solo quella croata/slovena.

0.1.

Tutte le forme raccolte vengono rappresentate con l'alfabeto fonetico internazionale, adattato come si usa, per ragioni tipografiche, nei lavori di questo tipo. Le forme che si riportano dalle fonti scritte rispettano la grafia originale.

L'accento viene segnato secondo i repertori più noti che riguardano le rispettive parlate. Da tener presente che i valori prosodici non sono identici per tutti gli idiomi. Per le forme slovene abbiamo usato il sistema a due unità di tipo non tonemico.

1. Elenco dei nomi popolari (le forme dalle fonti scritte si riconoscono facilmente dall'abbreviazione dell'opera):

Istroveneto: bocazo (Trieste/Trst - DORIA 80; KOS 62), legàj (Buie/Buje, Šverki), lègaj (Crassiza/Krasica, Montona/Motovun, Salvore/Savudrija, Umago/ Umag), lèganj (Santa Domenica/Labinci, Visignano/Višnjan), lègnjar (Pedena/Pićan), lègo (Canfanaro/Kanfanar), mràk (Crassiza/Krasica), nòtola (Bivio/Bivje), portaòvi (Visignano/Višnjan), šklepetàc (Torre/Tar), tetavache (Trst - DORIA 733; KOS 471; ROS 1151).

Istrioto: nòtola (Valle/Bale - CERN 74, "uccello notturno"), nòtula (Valle/Bale), šćokaàle (Gallesano/Galižana).

Istrorumeno: kragûju de nôpte (Sussgnevizza/Šušnjevica), lêgnjaru (Villanova/ Nova Vas), plôsko (Frassineto/Jesenovik), pû̀lju de nòpte (Seiane/Žejane). 
Croato: belulâska (Premci), kêgaj (Pračana), klepetâc (Bankovići, Gaiana/Gajana, Dignano/Vodnjan, Sbandati/Žbandaj), lẽga (Marzana/Marčana), lẽgaj (Krmed), lêgaj (Beletićev Brijeg, Foršići, Crassiza/Krasica, Lupoglav, Montona/Motovun, Pračana), lẽganj (Bersaz/Brseč), lêganj (Blaškovići, Monti/Breg, Ceppich/Čepić, Ičići, Pedena/Pićan, Sumberg/Šumber, Trget, Veli Golji), lềgalj (Valle/Bale), lêgnjar (Petehi), lèpuh (Brussich/Brušić), liêganj (Vermo/Beram, Katuni, Soldatići), lienjäk (Lindaro/Lindar), lîganj (Strmac), mrâk (Monpaderno/Baderna, Hreljići, Crassiza/Krasica, Ližnjan, Rovinjsko Selo, Valtura, Veli Vareški), nòćarica (Dobrigno/Dobrinj), nốni jastrebǐć (Promontore/Premantura), pâdovac (Pačići), plèhut (Dragosetti/Draguzeti), plôskavac (Kringa, Vela Traba), polegâj (Bergud piccolo/Mali Brgud) pologăjac (Munepiccolo/Male Mune, Munegrande/Vele Mune), vãjak (Katun), žâbar (Štrped).

Sloveno: galàt (Maresigo/Marezige), lahát (Gracischie/Gračišče), lúft (Covedo/ Kubed).

2.

All'inizio riteniamo opportuno riportare dai due repertori ornitologici i dati necessari per poter seguire meglio le spiegazioni delle denominazioni: "Velike njihove oči, mrko, nabuhlo perje kazuje nam jasno, da su to noćne ptice. Legnji imadu kratak kljun, vrlo duboko razciepljen; noge i prsti su im kratki (...) Oko širokih ralja imadu osobite, tvrde štetine, koje ždrielo još većim čine, za što mogu u lietu i veće noćne leptire loviti. U Evropi žive dvie vrste, od kojih je leganj, mračnjak, kozodoj, kozara, sisavac, pomrakuša, noćna lasta ili noćna lastavica, noćna ptica, mračna ptica, mračaj, mračnjak, sumračnik, legen, legnar, gluha, sojkula itd. (Caprimulgus europaeus ...) najviše raširen i kod nas. Ta je ptica sivo i smedje crtana i izšarana, koja boja posvema izgleda kao kora drveća, na kom se on kroz dan zadržaje (...) Kroz dan sjedi ili na zemlji, gdje se ne razlikuje svojom bojom od suhoga lišća ili na granama, ali u posve drugom položaju, nego druge ptice: ove sjede uviek popriečno, dok se legnji uzporedno sa granom polože. Gniezda ne prave nikakova. Dva bjelkasta jaja, što su izmrežkana crvenkastim crticama, snese ženka jednostavno na tle: na mahovinu ili na sušanj, najradije u grmlje." (GJURAŠIN I/258); "Grande come un Merlo, presenta grossa testa, becco piccolissimo, occhi grandi e zampe molto corte. Si mimetizza perfettamente sulla corteccia dei rami grazie al suo piumaggio dalle più svariate sfumature di marrone. Il $\sigma$ si riconosce per le macchie alari e per gli angoli posteriori della coda bianchi. $ᄋ$ e giovani non presentano chiazze chiare. Trascorre la giornata immobile, appollaiato tra i rami. $\mathrm{Nel}$ periodo riproduttivo canta soprattutto verso sera e di notte emettendo decrescenti 'trrr trrr'. Durante il volo le ali producono un caratteristico fruscio. Nidifica in foreste di confiere, in lande e in ambienti aridi. Epoca di nidificazione: V-VII, con due covate annue, composte da 2 uova ellittiche con marmoreggiature brune, che vengono deposte direttamente a terra." (DRCHAL 144).

3.

Il nostro è un uccello insignificante, come citato sopra durante la giornata rimane fermo su qualche ramo d'albero ed è molto difficile accorgersi della sua presenza 
(possiede una notevole capacità di mimicria). Praticamente non canta è nessuno lo mangia. Tutto ciò spiega più o meno pochi ornitonimi raccolti in Istria e quelli riportati nelle fonti scritte che riguardano il nostro territorio: ALI (4653) riporta le forme solamente per due località, mentre l'AIS e l'ASLEF non hanno la specie.

La tradizione latina segna caprimulgus (ANDR 48). André spiega la forma come calco linguistico greco $\alpha \gamma \gamma \sigma \vartheta \bar{\eta} \lambda \alpha \varsigma$ (traduzione ad litteram "chi succhia le capre"): "à tort, car cette formation se retrouve dans toutes les langues: fr. dial. tette chèvre, suisse rom. allaite tsivra, ital. succiacapre, gên. tetta crave, allemand Geismelker, Ziegenmelker, Kihmelker, angl. goat sucker, esp. chotacabras, etc."

Durante le nostre interviste in Istria non abbiamo segnato alcuna forma che esprimesse il sema citato. Nei repertori triestini troviamo tetavache, composto di teta (< titta "mammella", REW 8759) e vache (< vacca, REW 9109), nell' ALI, 4653, per Ajdovščina, kozodój, composto di koza "capra" (BEZLAJ IL/75; SKOK II/173) e doj(iti) "allattare" (BEZLAJ I/106; SKOK I/422). Il sema naturalmente non corrisponde alla realtà - ce lo spiega Salvadori: "Il nome di succiacapre è derivato a questi uccelli da ciò che posandosi frequentemente in mezzo agli armenti per cercare insetti nei loro escrementi, i pastori hanno creduto che succhiassero il latte alle capre." (SALVADORI, p. 48). ${ }^{1}$

\section{4.}

In Istria, nelle parlate croate e romanze, sono frequenti le denominazioni del tipo legaj (SKOK II/285) - gli ornitonimi romanzi di questo tipo sono prestiti croati. In alcuni paesi alla base lega $(n) j$ viene aggiunto il suffisso - $\operatorname{ar}$ (nella parlata istrorumena di Villanova -aru). Skok spiega l'ornitonimo lèganj (II/285) e forme simili come derivati del verbo lécí "covare". Bezlaj sottto il lemma legen (II/131) riporta la proposta etimologica di Skok aggiungendo però la riserva di Koštial. Propone anche la derivazione dalla radice indoeuropea *leg "tirare calci" (vedremo fra poco che anche questo sema ha dato un certo numero di denominazioni) e alla fine come conclusione lascia aperta la possibilità della fusione di due radici diverse.

Nonostante il comportamento diurno del nostro uccello possa senz'altro essere inteso come cova, alla lista degli etimi possibili per le forme del tipo legaj va aggiunto anche il verbo croatao ležati "giacere, star coricato". A favore di questa derivazione etimologica rivolgiamo l'attenzione alla forma lienjäk (Lindaro), se intesa come derivato dal croato lijen "pigro" (SKOK II/926) e polegâj (Bergud piccolo), dal ciacavo poležavati "starsene coricato un poco".

5.

Esprime probabilmente lo stesso semantismo anche l'ornitonimo pologäjac (Munepiccolo, Munegrande), un derivato del verbo croato polagati (jaja) "deporre (le uova)" - il succiacapre depone le uova direttamente sulla terra, senza fare il nido come gli altri uccelli.

1 Cfr. l'ittionimo negli Abruzzi succhja pècorë "Petromyzon marinus L." (JaFa 1.1.2.1.). 


\section{1.}

Il comportamento insolito nel deporre le uova ed il continuo spostamento di essa ha dato anche le seguenti denominazioni: istroven. portaòvi (Visignano), il sintagma dal verbo portar (< pŏrtare, REW 6672) e ovi, pl. del istroveneto ovo "uovo" (< ovum, REW 6128); ciacavo vãjak (Katun), un derivato nominale del verbo ciacavo va(l)jati "voltolare, rotolare" (SKOK III/563). L'ultima forma andrebbe nel paradigma degli ornitonimi che esprimono il sema "covare" se intesa come derivato del ciacavo valiti "covare" (SKOK III/563, s. v. valjatil).

6.

Il succiacapre è un uccello notturno. In otto località viene chiamato semplicemente mrâk (croato mrak "buio" - BEZLAJ II/199; SKOK II/467); a Dobrigno nöćarica (derivato in -ar + -ica del sostantivo noć "notte" (BEZLAJ II/226; SKOK II/522); a Sussgnevizza gli istrorumenofoni usano l'ornitonimo kragûju de nôpte, il sintagma di kraguju "Falco" e nopte "notte" (< nŏcte, REW 5973). L'ornitonimo istrorumeno combacia con quello croato di Promontore, nö́ni jastrebić, simile anche la denominazione a Seiane, pülju de nöpte, il sintagma dell'istrorum. pülju "uccello" (< pŭllus "animale giovane", DIV 865; REW 6828) e nöpte: "Po obrisu je podobna (sc. podhujka) sokolu." (GREGORI 185); "(...) njihov let (sc. ležetrudnikov) je podoben letu sokolov in lastovk." (BREHMS 225). A Valle, nella parlata istriota il succiacapre europeo viene denominato nòtula (Cernecca riporta la forma nòtola), a Bivio presso Capodistria nòtola (< *noctula "pipistrello", REW 5941.2). Le ultime tre forme citate nelle rispettive località vengono usate anche nel significato di "pipistrello" e perciò potrebbero appartenere al paradigma dei nomi che esprimono il sema "succhiare" - il pipistrello e noto tra la gente per la sua caratteristica di succhiare il sangue delle bestie nelle stalle, cosicche il transfer semantico "pipistrello" $\rightarrow$ "succiacapre" non dovrebbe stupire. Secondo noi però è più probabile che il transfer sia dovuto al carattere notturno comune all'uccello e al mammifero; da prendere in considerazione anche la somiglianza dei due volatili specialmente perché ambedue si vedono in volo soprattutto durante la notte.

7.

Il sema "il fruscio prodotto durante il volo" viene formalizzato negli ornitonimi di tipo klepetac, šklepetac (SKOK II/95) e ploskavac (SKOK II/691); cfr. lo slov. ploskati "applaudire": "Un rumoroso 'batter delle ali tra di loro' si ode frequentemente durante l'epoca delle cove." (DRCHAL 136).

I rumenofoni a Frassineto hanno storpiato la forma croata appena citata in plôsko la forma è interessante perché non esce in - $u$. Tutti gli ornitonimi finora citati di questo capitolo sono d'origine onomatopeica.

Va nello stesso paradigma l'ornitonimo plèhut (Dragosetti) ${ }^{2}$ e probabilmente anche il nome ciacavo lëpuh (Brussich) e quello sloveno lùft (Covedo) - dal tedesco Luft "aria". 
L'ornitimo istrioto šćokaàle è un sintagma dal verbo šćokar (triest. s'ciocar "schioccare, scoppiettare", DORIA 592) d'origine onomatopeica (DELI 1153: schioccàre "agitare, muovere in modo da produrre uno schiocco") e ale (pl. di ala < ala, REW 304). ${ }^{3}$

8.

L'andatura barcolante dell'uccello ("Zaradi izredno kratkih nog ne hodijo, pač pa zelo nerodno capljajo" - BREHMS 225) ha dato tre denominazioni. A Gracischie abbiamo segnato lahát. ${ }^{4} \mathrm{La}$ forma galàt (Maresigo) potrebbe essere spiegata con la metatesi $l$ - $h$ seguita dal cambiamento $h \rightarrow g$; nelle parlate slovene dell'Istria è comune un suono tra $g$ e $h$ (viene segnato con $\gamma$ ), esiste anche a Maresigo, cosicche il mutamento fonetico va spiegato in questo modo: $h \rightarrow \gamma \rightarrow g$.

L'ornitonimo pâdovac (Pačići), derivato in -ac del verbo padati "cadere" (SKOK IIU/615), è dovuto alla stessa caratteristica dell'uccello.

9.

Il sema "bocca grande" (cfr. la citazione dal Gjurašin all'inizio del saggio) viene formalizzato nell'ornitonimo bocazo (Trieste) riportato da Doria e Kosovitz, derivato in -azo dal triestino boca "bocca" (< bucca, REW 1357). ${ }^{5}$

La forma žâbar (Štrped), derivato in - ar dal croato žaba "rana" (SKOK IIU/668), come nome della specie Caprimulgus europaeus è isolato: "(...) il nome di calcabotto poi dal posarsi sulle strade quando verso sera vi saltellano piccoli rospi o botte, che alcuno ha creduto calcassero posandovisi sopra." (SALVADORI 48).

La forma belulâska (Premci) non ci è chiara.

\section{Abbreviazioni delle opere citate:}

AIS - Sprach-und Sachatlas Italiens und der Südschweitz, hgg. K. Jaberg und J. Judd, Zofingen 1928-40

ALI - Atlante Linguistico Italiano, materiale inedito consultabile all'Università degli Studi di Torino

ANDR - Jacques André, Les noms d'oiseaux en latin, Librairie C. Klincksieck, Parigi 1967

ASLEF - Atlante storico-linguistico-etnografico friulano, a cura di G. B. Pellegrini, Padova-Udine 1972

3 Anche Hirtz, p. 186, riporta due ornitonimi d'origine onomatopeica per questa specie che esprimono lo stesso sema: klek (in Konavle) e klepetavac (presso Neum e Klek).

4 Cfr. "láhati (...) 2. brzo hoditi uopće (podrugljivo) (...) U Vodicama s varijantom a - e lêhati (se), (...) 'amo-tamo tresti, gibati, njihati'...." (SKOK III/261).

5 Cfr. bokáš (Ajdovščina - ALI, 4653), derivato in -ă̌. 
BEZLAJ - F. Bezlaj, Etimološki slovar slovenskega jezika, I-II (A-O), SAZU, Lubiana 1977,1982

BREHMS - T. Jan, Brehm v barvah - Velika knjiga o živalih, Cankarjeva založba, Lubiana 1978

CERN(ECCA) - D. Cernecca, Dizionario del dialetto di Valle d'Istria, Centro di Ricerche Storiche Rovigno 1986

DELI - M. Cortelazzo - P. Zolli, Dizionario etimologico della lingua italiana, I-V, Zanichelli, Bologna 1979-1983

DIV - M. Divković, Latinsko - hrvatski rječnik za škole, Zagreb 1900 (ristampa dell'edizione del 1980)

DORIA - M. Doria, Grande dizionario del dialetto triestino, Edizioni "Italo Svevo" "Il Meridiano", Trieste 1984

DRCHAL - W. Černy - K. Drchal, Impariamo a conoscere gli uccelli, Istituto Geografico Deagostini, Novara 1982

GJURAŠIN - S. Gjurašin, Ptice, I-II, Naklada "Matice Hrvatske", Zagabria 1899, 1901

GREGORI - J. Gregori - I. Krečić, Naši ptiči, DZS, Lubiana 1979

HIRTZ - M. Hirtz, Rječnik narodnih zoologičkih naziva, JAZU, Zagabria 1938-1947

JaFa - V. Vinja, Jadranska fauna. Etimologija $i$ struktura naziva, I-II, JAZU-Logos, Zagabria-Spalato 1986

KOS - E. Kosovitz, Dizionario-vocabolario del dialetto triestino e della lingua italiana, Libreria internazionale "Italo Svevo", Trieste 1968

REW - W. Meyer-Lübke, Romanisches etymologisches Wörterbuch, Heidelberg 1972

ROS - E. Rosamani, Vocabolario giuliano, Capelli Editore, Bologna 1958

SALVADORI - T. Salvadori, Fauna d'Italia. Uccelli, Forni Editore, Bologna 1971 (ristampa dell'edizione dell' 1872)

SKOK - P. Skok, Etimologijski rječnik hrvatskoga ili srpskoga jezika, I-IV, JAZU, Zagabria 1971-1974

Povzetek

ISTRSKA ORNITONIMIJA: LJUDSKA IMENA ZA KOZODOJA (CAPRIMULGUS EUROPAEUS)

$V$ članku teče beseda o ljudskih ptičjih imenih za kozodoja (Caprimulgus europaeus) v Istri in na otoku Krku. Avtor je etimološko obdelal okoli 40 ornitonimov, ki jih je sam zbral v vseh istrskih govorih, slovanskih in romanskih. $\mathrm{Na}$ enak način je avtor obdelal tudi več ptičjih imen iz literature. 